\title{
A note on extended complex manifolds
}

\author{
S.A. Merkulov
}

\begin{abstract}
We introduce a category of extended complex manifolds, and prove that the functor describing deformations of a classical compact complex manifold $M$ within this category is versally representable by (an analytic subspace in) $\mathbf{H}^{*}\left(M, T_{M}\right)$.

By restricting the associated versal family of extended complex manifolds over $\mathbf{H}^{*}\left(M, T_{M}\right)$ to the subspace $\mathbf{H}^{1}\left(M, T_{M}\right)$ one gets a correct limit to the classical picture.
\end{abstract}

0. Introduction. Recent advances in homological mirror symmetry [Ba, BK, Ko1, Ma1, Ma2, P, PZ give a strong evidence for the existence of extended versions of at least three classical categories in algebraic geometry - the categories of compact complex manifolds, of coherent sheaves, and of submanifolds. One such category (of extended special Lagrangian submanifolods) has been constructed in [Me1]. In this note we suggest an extension of the category of complex manifolds.

Curiously, even with no appropriate notion of extended complex structure at hand, one can nevertheless say a lot about their moduli spaces [Ba, BK, Ma1, Me2]. This eggbefore-chicken situation is due to a remarkably effective, purely algebraic paradigm of the modern deformation theory (see GM, Ko2 and references therein):

- Given a mathematical structure one wishes to deform, the first step should be a search for a differential $\mathbb{Z}$-graded (dg-, for short) Lie $k$-algebra $\left(\mathfrak{g}=\bigoplus_{i \in \mathbb{Z}} \mathfrak{g}^{i}, d,[],\right)$ which "controls" the deformations. Next one defines a deformation functor

$$
\operatorname{Def}_{\mathfrak{g}}^{0}:\left\{\begin{array}{l}
\text { the category of Artin } \\
k \text {-local algebras }
\end{array}\right\} \longrightarrow\{\text { the category of sets }\}
$$

as follows

$$
\operatorname{Def}_{\mathfrak{g}}^{0}(A)=\left\{\Gamma \in\left(\mathfrak{g} \otimes m_{A}\right)^{1} \mid d \Gamma+\frac{1}{2}[\Gamma, \Gamma]=0\right\} / \exp \left(\mathfrak{g} \otimes m_{A}\right)^{0},
$$

where $m_{A}$ is the maximal ideal of the Artin algebra $A$, the latter is viewed as a $\mathbb{Z}$-graded algebra concentrated in degree zero (so that $\left(\mathfrak{g} \otimes m_{\mathcal{A}}\right)^{i}=\mathfrak{g}^{i} \otimes m_{A}$ ), and the quotient is taken with respect to the following representation of the gauge group $\exp \left(\mathfrak{g} \otimes m_{A}\right)^{0}$,

$$
\Gamma \rightarrow \Gamma^{g}=e^{\operatorname{ad}_{g}} \Gamma-\frac{e^{a d_{g}}-1}{\operatorname{ad}_{g}} d g, \quad g \in\left(\mathfrak{g} \otimes m_{A}\right)^{0},
$$


where ad is just the usual internal automorphism of $\mathfrak{g}, \operatorname{ad}_{g} \Gamma:=[g, \Gamma]$. Finally one tries to represent the deformation functor by a topological (pro-Artin) algebra $\mathcal{O}_{S}$ so that

$$
\operatorname{Def}_{\mathfrak{g}}^{0}(A)=\operatorname{Hom}_{\text {cont }}\left(\mathcal{O}_{S}, A\right) .
$$

This associates to the given mathematical structure a formal moduli space $S$ whose "ring of functions" is $\mathcal{O}_{S}$.

The tangent space, $\operatorname{Def}_{\mathfrak{g}}^{0}\left(k[\varepsilon] / \varepsilon^{2}\right)$, to the functor $\operatorname{Def}_{\mathfrak{g}}^{0}$ is isomorphic to the cohomology group $\mathbf{H}^{1}(\mathfrak{g})$ of the complex $(\mathfrak{g}, d)$. If one extends in the obvious way the above deformation functor to the category of arbitrary $\mathbb{Z}$-graded $k$-local Artin algebras (which may not be concentrated in degree 0), one gets the functor Def $\mathfrak{g}_{\mathfrak{g}}^{*}$ with the tangent space isomorphic to the full cohomology group $\mathbf{H}^{*}(\mathfrak{g})=\bigoplus_{i \in \mathbb{Z}} \mathbf{H}^{i}(\mathfrak{g})$.

The dg-Lie algebra controlling deformations of a given complex structure on a compact manifold $M$ is $\left(\mathfrak{g}:=\bigoplus_{i} \Gamma\left(M, T_{M} \otimes \Omega_{M}^{0, i}\right), \bar{\partial}\right)$, where $T_{M}$ is the tangent holomorphic sheaf, and $\Omega_{M}^{0, i}$ the sheaf of $(0, i)$ forms. The classical deformation functor $\operatorname{Def}_{\mathfrak{g}}^{0}$ is known to be versally representable by the Kuranishi analytic subspace in $\mathbf{H}^{1}(\mathfrak{g})=\mathbf{H}^{1}\left(M, T_{M}\right)$. Its extension, Def ${ }_{\mathfrak{g}}^{*}$, is the main technical tool for introducing and studying the moduli space of so called extended complex structures [BK]. Actually, the authors of [BK] go even further and study the extended deformation functor associated with the larger dg-Lie algebra, $(\hat{\mathfrak{g}}=$ $\left.\Gamma\left(M, \wedge^{*} T_{M} \otimes \Omega_{M}^{0, *}\right), \bar{\partial}\right)$. They prove that $\operatorname{Def}_{\hat{\mathfrak{g}}}^{*}$ is non-obstructed provided $M$ is a Calabi-Yau manifold, and show that the associated extended moduli space, $S_{\text {ext }} \simeq \mathbf{H}^{*}\left(M, \wedge^{*} T_{M}\right)$, has an induced structure of Frobenius manifold [BK, Ba]. For arbitrary $M$, the moduli space $S_{\text {ext }}$ is canonically an $F_{\infty}$-manifold Me2].

There is, however, an obvious problem with the above approach as it offers no geometric explanation of what this extended complex structure might be. It is an urgent and important problem Ma2 to find a geometric embodiment of this notion, develop its deformation theory, and check that the base of the resulting versal deformation can be canonically identified with what one gets from the above purely algebraic approach. The present note offeres a realization of this programme for the extended deformation functor Def $_{\mathfrak{g}}^{*}$. It is unlikely, however, that the same approach will yield a geometrical model for the functor $\operatorname{Def}_{\mathfrak{g}}^{*}$ — this seems to be a much more intricate object.

1. Extended complex manifolds. These will be defined in two steps. First comes the notion of pre-complex manifold.

1.1. Model pre-complex structures. Let $U$ be an open domain in $\mathbb{R}^{2 n \mid 0}$. A pre-complex structure on $U$ is a map

$$
\phi: U \longrightarrow \mathbb{C}^{2 n \mid n}
$$

such that

(i) $\phi$ is a smooth embedding, and

(ii) at each $x \in U$, the completions of the associated stalks of the sheaves $C^{\infty}(U)$ and $\phi^{-1}\left(\mathcal{O}_{\mathbb{C}^{2 n \mid n}} /\right.$ nilpotents $)$ are isomorphic. 
Here $C^{\infty}(U)$ stands for the sheaf of smooth complex valued functions on $U$, and $\mathcal{O}_{\mathbb{C}^{2 n \mid n}}$ for the sheaf of holomorphic functions on the supermanifold $\mathbb{C}^{2 n \mid n}$. The ringed space

$$
\mathcal{U}^{n}=\left(U, \mathcal{O}_{U}^{\text {pre }}:=\phi^{-1}\left(\mathcal{O}_{\mathbb{C}^{2 n \mid n}}\right)\right)
$$

is called a pre-complex domain of dimension $n$. The reduced sheaf $\mathcal{O}_{U}^{\text {pre }} /$ nilpotents is denoted sometimes by $\mathcal{O}_{U, \text { rd }}^{\text {pre }}$.

A morphism of pre-complex domains $\mathcal{U}^{n} \rightarrow \mathcal{V}^{m}$ is, by definition, a pair $\left(f_{C^{\infty}},[f]\right)$, consisting of a smooth map $f_{C^{\infty}}: U \rightarrow V$ and a germ, $[f]$, of holomorphic maps $f$ from a small open neighbourhood of $\operatorname{Im}\left(\phi_{U}\right)$ in $\mathbb{C}^{2 n \mid n}$ to a small open neighbourhood of $\operatorname{Im}\left(\phi_{V}\right)$ in $\mathbb{C}^{2 m \mid m}$ such that, for each $x \in U$,

$$
f_{C^{\infty}}^{*}\left(\overline{\overline{\mathcal{O}_{V, \mathrm{rd}, x}^{\mathrm{pre}}}}\right)=\overline{\overline{f^{*}\left(\mathcal{O}_{V, \mathrm{rd}, x}^{\mathrm{pre}}\right)}},
$$

where $=$ stands for the completion of the stalks at $x$ with respect to the natural ideals. We often abbreviate $\left(f_{C^{\infty}},[f]\right)$ simply to $f$.

1.2. Fact. Any open domain $U \subset \mathbb{R}^{2 n \mid 0}$ admits a pre-complex structure. Indeed, identifying $\mathbb{R}^{2 n \mid 0}$ with $\mathbb{C}^{n \mid 0}$ one immediately gets a required map $\phi$ by further identifying $C^{n \mid 0}$ with the "diagonal" subspace in $\mathbb{C}^{2 n \mid n}$ given, in the natural coordinates $\left(z^{\alpha}, z^{\dot{\alpha}}, \psi^{\dot{\alpha}}\right)$, $\alpha, \dot{\alpha}=1, \ldots, n$, on $\mathbb{C}^{2 n \mid n}$, by the equations

$$
z^{\dot{\alpha}}=\overline{\left(z^{\alpha}\right)}, \quad \psi^{\dot{\alpha}}=0,
$$

where the bar denotes complex conjugation.

1.3. Definition. An $n$-dimensional pre-complex manifold is a ringed space $\mathcal{M}=$ $\left(M, \mathcal{O}_{M}^{\text {pre }}\right)$ modeled on pre-complex domains of dimension $n$. It is called compact if the underlying smooth manifold $M$ is compact.

A holomorphic vector field $v$ on $\mathcal{M}$ is, as usual, a $\mathbb{C}$-linear automorphism of the structure sheaf,

$$
v: \mathcal{O}_{M}^{\text {pre }} \longrightarrow \mathcal{O}_{M}^{\text {pre }}
$$

whose restriction to each stalk, $\mathcal{O}_{M, x}^{\text {pre }}$, is a derivation of the $\operatorname{ring} \mathcal{O}_{M, x}^{\text {pre }}$. The sheaf of holomorphic vector fields on $\mathcal{M}$ is denoted by $\mathcal{T}_{\mathcal{M}}$.

1.4. Definition. An extended complex manifold is a pair $\left(\mathcal{M}, \partial_{\mathcal{M}}\right)$ consisting of a compact pre-complex manifold $\mathcal{M}$ and an odd holomorphic vector field $\partial_{\mathcal{M}}$ such that $\left[\partial_{\mathcal{M}}, \partial_{\mathcal{M}}\right]=0$ 吅 and $\operatorname{dim} \mathbf{H}\left(\mathcal{M}, \partial_{\mathcal{M}}\right)<\infty$. Here

$$
\mathbf{H}\left(\mathcal{M}, \partial_{\mathcal{M}}\right):=\frac{\Gamma\left(M, \operatorname{Ker}\left[\partial_{\mathcal{M}}, \ldots\right]\right)}{\Gamma\left(M, \operatorname{Im}\left[\partial_{\mathcal{M}}, \ldots\right]\right)}
$$

with the differential given by

$$
\begin{aligned}
{\left[\partial_{\mathcal{M}}, \ldots\right]: \mathcal{T}_{\mathcal{M}} } & \longrightarrow \mathcal{T}_{\mathcal{M}} \\
V & \longrightarrow\left[\partial_{\mathcal{M}}, V\right] .
\end{aligned}
$$

\footnotetext{
${ }^{1}$ Odd vector fields with this property are often called homological.
} 
Note that $\mathbf{H}\left(\mathcal{M}, \partial_{\mathcal{M}}\right)$ is canonically a Lie superalgebra with the brackets, $[$, ] $\mathbf{H}$, induced from the usual commutator of holomorphic vector fields.

One can also associate with $\left(\mathcal{M}, \partial_{\mathcal{M}}\right)$ the cohomology manifold, $\mathcal{H}\left(\mathcal{M}, \partial_{\mathcal{M}}\right)$, which is, by definition, a ringed space $\left(M, \operatorname{Ker} \partial_{\mathcal{M}} / \operatorname{Im} \partial_{\mathcal{M}}\right)$.

A morphism of extended complex manifolds,

$$
f:\left(\mathcal{M}, \partial_{\mathcal{M}}\right) \longrightarrow\left(\mathcal{S}, \partial_{\mathcal{S}}\right)
$$

is a morphism of the associated pre-complex manifolds, $f:\left(M, \mathcal{O}_{M}^{\text {pre }}\right) \rightarrow\left(S, \mathcal{O}_{M}^{\text {pre }}\right)$, which commutes with the homological vector fields, i.e.

$$
\partial_{\mathcal{M}}\left(f^{*}(g)\right)=f^{*}\left(\partial_{\mathcal{S}} g\right)
$$

for any $g \in \mathcal{O}_{S}^{\text {pre }}$. One may reformulate this as $f_{*}\left(\partial_{\mathcal{M}}\right)=\partial_{\mathcal{S}}$.

1.5. Basic example. Let $M$ be a compact complex manifold. There is associated an extended complex manifold $\left(\mathcal{M}=\left(M, \mathcal{O}_{M}^{\text {pre }}\right), \bar{\partial}\right)$ constructed as follows:

(i) First consider a natural embedding,

$$
\begin{aligned}
\phi: M & \longrightarrow M \times \Pi T_{\bar{M}} \\
x & \longrightarrow(x, O(x))
\end{aligned}
$$

where $T_{\bar{M}}$ is the total space of the holomorphic vector bundle over the conjugate complex manifold $\bar{M}$, $\Pi$ the parity change functor, and $O: \bar{M} \rightarrow \Pi T_{\bar{M}}$ the zero section.

(ii) Set

$$
\mathcal{O}_{M}^{\text {pre }}:=\phi^{-1}\left(\text { the structure sheaf on } M \times \Pi T_{\bar{M}}\right) \text {. }
$$

(iii) Note that $\Pi T_{\bar{M}}$ is canonically a dg-manifold (of dimension $n \mid n$ ) with the homological field being just the $(1,0)$-part of the de Rham differential on $\bar{M}$. The latter induces a homological vector field on $\mathcal{M}$ which we denote by $\bar{\partial}$.

1.5.1. Remark. Let $T_{M}$ be the holomorphic tangent sheaf on $M$. We make the cohomology $\mathbf{H}^{*}\left(M, T_{M}\right)=\bigoplus_{k} \mathbf{H}^{k}\left(M, T_{M}\right)$ into a superspace by setting

$$
\mathbf{H}^{*}\left(M, T_{M}\right)_{\tilde{0}}=\bigoplus_{k \text { is odd }} \mathbf{H}^{k}\left(M, T_{M}\right), \quad \mathbf{H}^{*}\left(M, T_{M}\right)_{\tilde{1}}=\bigoplus_{k \text { is even }} \mathbf{H}^{k}\left(M, T_{M}\right) .
$$

This choice of $\mathbb{Z}_{2}$-grading is in agreement with the classical deformation theory where $\mathbf{H}^{1}\left(M, T_{M}\right)$ is even. Then the natural map,

$$
\begin{aligned}
& T_{M} \otimes \Omega_{M}^{0, p} \times T_{M} \otimes \Omega_{M}^{0, q} \quad \longrightarrow \quad T_{M} \otimes \Omega_{M}^{0, p+q} \\
& X \otimes \alpha \quad \times \quad Y \otimes \beta \quad \longrightarrow \quad[X, Y] \otimes(\alpha \wedge \beta)
\end{aligned}
$$

induces on $\mathbf{H}^{*}\left(M, T_{M}\right)$ the structure of an odd Lie superalgebra (cf. Ma1). Reversing the parity, we make $\Pi \mathbf{H}^{*}\left(M, T_{M}\right)$ into a Lie superalgebra.

1.5.2. Lemma. Let $M$ be a complex manifold, and $(\mathcal{M}, \bar{\partial})$ the associated extended complex manifold. Then 
(a) $\mathcal{H}(\mathcal{M}, \bar{\partial})$ is precisely $M$ with its original complex structure.

(b) $\mathbf{H}(\mathcal{M}, \bar{\partial})=\Pi \mathbf{H}^{*}\left(M, T_{M}\right)$ as Lie superalgebras.

Proof. The statement (a) follows immediately from the Poincare $\bar{\partial}$-lemma. The second statement requires a small computation. Let $\left\{z^{\alpha}\right\}$ be a local coordinate system on $M$, and $\left\{z^{\dot{\alpha}}, \psi^{\dot{\alpha}}=d z^{\dot{\alpha}}\right\}$ the associated local coordinate system on $\Pi T_{\bar{M}}$. The collection $\left\{z^{\alpha}, z^{\dot{\alpha}}, \psi^{\dot{\alpha}}\right\}$ gives rise to a coordinate chart on $\left(M, \mathcal{O}_{M}^{\text {pre }}\right)$ so that any $V \in \Gamma\left(M, \mathcal{T}_{\mathcal{M}}\right)$ can be locally represented as

$$
V=\sum_{\alpha=1}^{n} V^{\alpha} \frac{\partial}{\partial z^{\alpha}}+\sum_{\dot{\alpha}=1}^{n}\left(V^{\dot{\alpha}} \frac{\partial}{\partial z^{\dot{\alpha}}}+W^{\dot{\alpha}} \frac{\partial}{\partial \psi^{\dot{\alpha}}}\right),
$$

for some local sections, $V^{\alpha}, V^{\dot{\alpha}}, W^{\dot{\alpha}}$, of $\mathcal{O}_{M}^{\text {pre }}$. As

$$
\bar{\partial}=\sum_{\dot{\alpha}=1}^{n} \psi^{\dot{\alpha}} \frac{\partial}{\partial z^{\dot{\alpha}}}
$$

we have

$$
\left[\bar{\partial}_{0}, V\right]=\sum_{\alpha=1}^{n}\left(\bar{\partial} V^{\alpha}\right) \frac{\partial}{\partial z^{\alpha}}+\sum_{\dot{\alpha}=1}^{n}\left(\bar{\partial} V^{\dot{\alpha}}-(-1)^{\tilde{V}} W^{\dot{\alpha}}\right) \frac{\partial}{\partial z^{\dot{\alpha}}}+\sum_{\dot{\alpha}=1}^{n}\left(\bar{\partial} W^{\dot{\alpha}}\right) \frac{\partial}{\partial \psi^{\dot{\alpha}}} .
$$

Thus $V \in \Gamma(M, \operatorname{Ker}[\bar{\partial}, \ldots])$ if and only if $\bar{\partial} V^{\alpha}=0, W^{\dot{\alpha}}=(-1)^{\tilde{V}} \bar{\partial} V^{\dot{\alpha}}$ and $V^{\dot{\alpha}}$ arbitrary. Moreover, the equivalence class $V \bmod \Gamma(M, \operatorname{Im}[\bar{\partial}, \ldots])$ always has a representative of the form

$$
\sum_{\alpha=1}^{n} V^{\alpha} \frac{\partial}{\partial z^{\alpha}}
$$

where $V^{\alpha}$ are defined uniquely up to addition of a $\bar{\partial}$-exact term. The Dolbeault theorem completes the proof.

2. Deformation theory. We start with the deformation theory of dg-manifolds and then apply the technique to extended complex manifolds.

2.1. Dg-manifolds. An extended complex manifold is a particular case of a complex differential $\mathbb{Z}_{2}$-graded (dg-, for short) manifold which is, by definition, a complex supermanifold equipped with a homological holomorphic vector field (cf. [Ko2]). Morphisms of dg-manifolds,

$$
\left(\mathcal{X}, \partial_{\mathcal{X}}\right) \stackrel{f}{\longrightarrow}\left(\mathcal{S}, \partial_{\mathcal{S}}\right)
$$

are defined as in sect. 1.4. It is easy to see that the resulting category is closed with respect to the fibered products. Note that the fibres of $f$ are not, in general, dg-manifolds except over the points where $\partial_{\mathcal{S}}$ vanishes. In this context we define a pointed dg-manifold as a triple $\left(\mathcal{S}, \partial_{\mathcal{S}}, *\right)$, where $\left(\mathcal{S}, \partial_{\mathcal{S}}\right)$ is a (formal) dg-manifold and $*$ a point in $\mathcal{S}$ such that $\partial_{\mathcal{S}} I_{*} \subset I_{*}^{2}, I_{*}$ being the ideal sheaf of $*$. 
2.1.1. Remark. According to Kontsevich [Ko2], any Lie superalgebra structure, [, ], on a vector superspace $\mathfrak{g}$ can be equivalently interpreted as a quadratic homological vector field on $(\Pi \mathfrak{g}, 0)$ viewed as a pointed formal supermanifold. Thus $\mathbf{H}^{*}\left(M, T_{M}\right)$ in example 1.5, and $\Pi \mathbf{H}\left(\mathcal{M}, \partial_{\mathcal{M}}\right)$ in 1.4 are canonically pointed dg-manifolds.

2.1.2. Lemma. Let $\left(\mathcal{S}, \partial_{\mathcal{S}}, *\right)$ be a pointed dg-manifold. Then $\Pi \mathcal{T}_{\mathcal{S}, *}$, the tangent space at $*$ with the reversed parity, is canonically a Lie superalgebra.

Proof. Let $v_{1}$ and $v_{2}$ be two tangent vectors at $* \in \mathcal{S}$, and $V_{1}$ and $V_{2}$ any two germs of holomorphic vector fields such that $\left.V_{1}\right|_{*}=v_{1}$ and $\left.V_{2}\right|_{*}=v_{2}$. The bilinear skew-symmetric operation,

$$
\begin{aligned}
& {[,]_{*}: \Pi \mathcal{T}_{\mathcal{S}, *} \times \Pi \mathcal{T}_{\mathcal{S}, *} \longrightarrow \quad \Pi T_{\mathcal{S}, *}} \\
& \Pi v_{1} \times\left.\Pi v_{2} \longrightarrow \Pi\left[V_{1},\left[\partial_{\mathcal{S}}, V_{2}\right]\right]\right|_{*}
\end{aligned}
$$

is well defined. The identity $\left[\partial_{\mathcal{S}}, \partial_{\mathcal{S}}\right]=0$ implies the Jacobi identity for $[,]_{*}$.

2.2. Deformations. A deformation of a complex dg-manifold $\left(\mathcal{M}, \partial_{\mathcal{M}}\right)$ is, by definition, a morphism,

$$
\left(\mathcal{X}, \partial_{\mathcal{X}}\right) \stackrel{f}{\longrightarrow}\left(\mathcal{S}, \partial_{\mathcal{S}}, *\right)
$$

from a complex dg-manifold $\left(\mathcal{X}, \partial_{\mathcal{X}}\right)$ to a pointed complex dg-base $\left(\mathcal{S}, \partial_{\mathcal{S}}, *\right)$ such that

(a) $\left(f^{-1}(*),\left.\partial_{\mathcal{X}}\right|_{f^{-1}(*)}\right) \stackrel{i}{\simeq}\left(\mathcal{M}, \partial_{\mathcal{M}}\right)$, and

(b) the associated morphism of complex supermanifolds, $f: \mathcal{X} \rightarrow \mathcal{S}$ is locally trivial in an neighbourhood of $* \in S$.

Given two deformations, $f:\left(\mathcal{X}, \partial_{\mathcal{X}}\right) \rightarrow\left(\mathcal{S}, \partial_{\mathcal{S}}, *\right)$ and $\tilde{f}:\left(\tilde{\mathcal{X}}, \partial_{\tilde{\mathcal{X}}}\right) \rightarrow\left(\tilde{\mathcal{S}}, \partial_{\tilde{\mathcal{S}}}, *\right)$, of the same dg-manifold manifold $\mathcal{M}$, a morphism from the first to the second, is, by definition, a commutative diagram

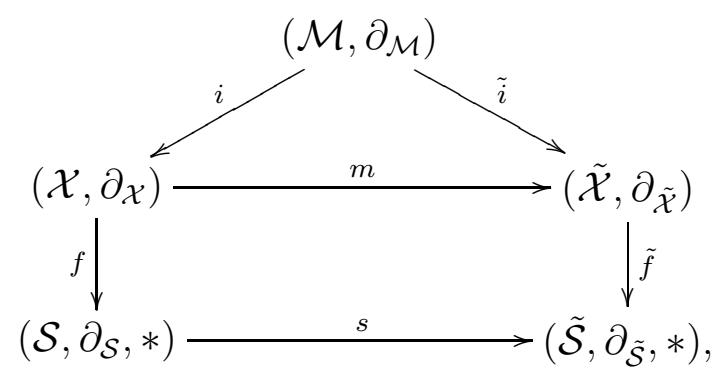

where $m$ (resp. $s$ ) is a morphism of (resp. pointed) dg-manifolds. If $s$ is the identity morphism and $m$ is an isomorphism, then the resulting morphism is called an equivalence of deformations.

As usually, one defines a deformation of a smooth dg-manifold over a germ of pointed (possibly, singular) dg-bases. From now on we consider only such deformations.

2.3. Remark. It may look puzzling that we allow in defintion 2.2 the homological vector field $\partial_{\mathcal{S}}$ to be non-zero - the fibres of $f$ over the points where $\partial_{\mathcal{S}} \neq 0$ are not, in general, dg-manifolds. A more natural definition of deformation would be a version of 2.2 with $\partial_{\mathcal{S}} \equiv 0$, where the base is simply a pointed analytic superspace (let us term these 0 -deformations). There are two advantages with understanding a deformation as in 2.2 : 
(i) By allowing $\partial_{\mathcal{S}} \neq 0$, we do not loose generality; 0-deformation is a deformation.

(ii) The 0-deformation theory of $\left(\mathcal{M}, \partial_{\mathcal{M}}\right)$ is, in general, obstructed, and its versal moduli space $\left(\mathcal{V}_{\text {versal }}, *\right)$, when it exists, is a singular analytic space. The role of $\partial_{\mathcal{S}}$ in 2.2 is to overcome all these obstructions producing thereby a smooth versal moduli dgspace, $\left(\mathcal{S}, \partial_{\mathcal{S}}, *\right)$, of which $\mathcal{V}_{\text {versal }}$ is merely a (singular) analytic subspace given by the equations $\partial_{\mathcal{S}}=0$ (cf. [Me2]). In a sense, the deformation theory 2.2 is a smooth resolution of the more natural 0-deformation theory.

2.4. Proposition. Let $f:\left(\mathcal{X}, \partial_{\mathcal{X}}\right) \rightarrow\left(\mathcal{S}, \partial_{\mathcal{S}}, *\right)$ be a deformation of a dg-manifold $\left(\mathcal{M}, \partial_{\mathcal{M}}\right)$. There is a canonical (even) morphism of Lie superalgebras,

$$
D f:\left(\Pi \mathcal{T}_{\mathcal{S}, *},[,]_{*}\right) \longrightarrow\left(\mathbf{H}\left(\mathcal{M}, \partial_{\mathcal{M}}\right),[,]_{\mathbf{H}}\right) \text {. }
$$

Proof. Let is first construct an odd linear morphism,

$$
d f: \mathcal{T}_{\mathcal{S}, *} \longrightarrow \mathbf{H}\left(\mathcal{M}, \partial_{\mathcal{M}}\right)
$$

of vector superspaces. Fixing a local trivialization, $\phi: \mathcal{X} \simeq \mathcal{S} \times \mathcal{M}$, we may decomposef?

$$
\partial_{\mathcal{X}}=\partial_{\mathcal{M}}+\partial_{\mathcal{S}}+\Gamma
$$

for some $f$-vertical odd holomorphic field $\Gamma$ which vanishes at $\mathcal{M} \times *$. Then the homology condition $\left[\partial_{\mathcal{X}}, \partial_{\mathcal{X}}\right]=0$ translates into the Maurer-Cartan(-like) equations,

$$
\left[\partial_{\mathcal{M}}, \Gamma\right]+\left[\partial_{\mathcal{S}}, \Gamma\right]+\frac{1}{2}[\Gamma, \Gamma]=0
$$

For a $v$ in $\mathcal{T}_{\mathcal{S}, *}$, we define a global holomorphic vector field on $\mathcal{M}$,

$$
d f(v):=\left.[V, \Gamma]\right|_{f^{-1}(*)}
$$

where $V$ is an arbitrary extension of $v$ to a germ of holomorphic vector field on $(\mathcal{S}, *)$. The Maurer-Cartan equations and the fact that $\partial_{\mathcal{S}}$ has zero at $*$ of second order imply

$$
\left[\partial_{\mathcal{M}}, d f(v)\right]=0
$$

Moreover, the cohomology class of $d f(v)$ in $\mathbf{H}\left(\mathcal{M}, \partial_{\mathcal{M}}\right)$ does not depend on the choice of the trivialization $\phi$ so that the map $d f$ is well-defined.

Analogously, for any $v_{1}, v_{2} \in \mathcal{T}_{\mathcal{S}, *}$ we have

$$
\left[V_{1},\left[V_{2},\left[\partial_{\mathcal{M}}, \Gamma\right]\right]\right]+\left[V_{1},\left[V_{2},\left[\partial_{\mathcal{S}}, \Gamma\right]\right]\right]+\frac{1}{2}\left[V_{1},\left[V_{2},[\Gamma, \Gamma]\right]\right]=0
$$

implying

$$
\left.\left[\left[V_{1},\left[\partial_{\mathcal{S}}, V_{2}\right]\right], \Gamma\right]\right|_{f^{-1}(*)}+\left[\left.\left[V_{1}, \Gamma\right]\right|_{f^{-1}(*)},\left.\left[V_{2}, \Gamma\right]\right|_{f^{-1}(*)}\right]=0 \bmod \operatorname{Im}\left[\partial_{\mathcal{M}}, \ldots\right] .
$$

Thus $D f:=d f \Pi$ is a morphism of Lie superalgebras.

\footnotetext{
${ }^{2}$ We apologize for being a bit sloppy in formulating this decomposition.
} 
2.5. Versality. If $f:\left(\mathcal{X}, \partial_{\mathcal{X}}\right) \rightarrow\left(\mathcal{S}, \partial_{\mathcal{S}}, *\right)$ is a deformation of a dg-manifold $\left(\mathcal{M}, \partial_{\mathcal{M}}\right)$, and $g:\left(\tilde{\mathcal{S}}, \partial_{\tilde{\mathcal{S}}}, *\right) \rightarrow\left(\mathcal{S}, \partial_{\mathcal{S}}, *\right)$ is a morphism of germs of pointed dg-spaces, then the fibred product,

$$
\left(\mathcal{X}, \partial_{\mathcal{X}}\right) \times_{\left(\mathcal{S}, \partial_{\mathcal{S}}\right)}\left(\tilde{\mathcal{S}}, \partial_{\tilde{\mathcal{S}}}\right)
$$

gives rise to the induced deformation, $g^{*}(f)$, of $\mathcal{M}$ over the germ $\left(\tilde{\mathcal{S}}, \partial_{\tilde{\mathcal{S}}}, *\right)$.

A deformation $f:\left(\mathcal{X}, \partial_{\mathcal{X}}\right) \rightarrow\left(\mathcal{S}, \partial_{\mathcal{S}}, *\right)$ is called versal if every other deformation $f:\left(\tilde{\mathcal{X}}, \partial_{\tilde{\mathcal{X}}}\right) \rightarrow\left(\tilde{\mathcal{S}}, \partial_{\tilde{\mathcal{S}}}, *\right)$ of the same extended complex manifold is equivalent to the inverse image, $g^{*}(f)$, of $f$ under some morphism of germs, $g:\left(\tilde{\mathcal{S}}, \partial_{\tilde{\mathcal{S}}}, *\right) \rightarrow\left(\mathcal{S}, \partial_{\mathcal{S}}, *\right)$, of pointed dg-spaces. A versal deformation $f$ is called minimal if the associated morphism of Lie superalgebras $D f$ (see Proposition 2.4) is an isomorphism. Any two minimal versal deformations of $\left(\mathcal{M}, \partial_{\mathcal{M}}\right)$ are isomorphic.

2.6. Theorem. Let $\left(\mathcal{M}, \partial_{\mathcal{M}}\right)$ be a dg-manifold with $\operatorname{dim} \mathbf{H}\left(\mathcal{M}, \partial_{\mathcal{M}}\right)<\infty$. Then there exists a smooth minimal versal deformation of $\left(\mathcal{M}, \partial_{\mathcal{M}}\right)$.

Proof. This is a special case of the Smoothness Theorem 2.5.6 in Me2].

2.7. Example. To any (compact) smooth manifold $M$ one may associate a dgmanifold,

$$
\left(\mathcal{M}=\Pi T_{M}, \partial_{\mathcal{M}}=\right.\text { de Rham differential). }
$$

As $\mathbf{H}\left(\mathcal{M}, \partial_{\mathcal{M}}\right)$ always vanishes (an easy exercise), this dg-manifold is rigid, i.e. its any deformation is trivial. This is in accord with the topological nature of the example.

2.7. Deformation theory of extended complex manifolds. This is an obvious modification of the deformation theory of dg-manifolds with all the notions and results from 2.1-2.6 holding true. For example, a deformation of an extended complex manifold $\left(\mathcal{M}, \partial_{\mathcal{M}}\right)$ is a proper morphism of complex dg-manifolds,

$$
\left(\mathcal{X}, \partial_{\mathcal{X}}\right) \stackrel{f}{\longrightarrow}\left(\mathcal{S}, \partial_{\mathcal{S}}, *\right)
$$

such that

(a) for each $t \in \operatorname{Zeros}\left(\partial_{\mathcal{S}}\right)$ the fibre $\left(f^{-1}(t),\left.\partial_{\mathcal{X}}\right|_{f^{-1}(t)}\right)$ is an extended complex manifold,

(b) $\left(f^{-1}(*),\left.\partial_{\mathcal{X}}\right|_{f^{-1}(*)}\right) \stackrel{i}{\simeq}\left(\mathcal{M}, \partial_{\mathcal{M}}\right)$, and

(c) the associated morphism of complex supermanifolds, $f: \mathcal{X} \rightarrow \mathcal{S}$ is locally trivial in an neighbourhood of $* \in S$.

2.7.1. Remarks. (i) In view of 1.1(ii), the local triviality condition 2.7(c) ensures that the underlying smooth structure of $\mathcal{M}$ keeps unchanged upon deformation. It is only the homological vector field that undergoes deformation.

(ii) The induced morphism, $f: \mathcal{H}\left(\mathcal{X}, \partial_{\mathcal{X}}\right) \rightarrow\left(\mathcal{H}\left(\mathcal{S}, \partial_{\mathcal{S}}\right)\right.$, $\left.*\right)$, may not be locally trivial.

2.7.2. Theorem. Let $M$ be a compact complex manifold and $(\mathcal{M}, \bar{\partial})$ the associated extended complex manifold. Then there exists a minimal versal deformation of the latter of the form

$$
\left(\mathcal{X}, \partial_{\mathcal{X}}\right) \stackrel{f}{\longrightarrow}\left(\mathbf{H}^{*}\left(M, T_{M}\right), \partial, 0\right)
$$

Moreover, 
(i) the homological vector field $\partial$ is an invariant of the original complex manifold $M$.

(ii) The restriction of the family $f$ to the analytic subspace $\operatorname{Zeros}(\partial) \cap \mathbf{H}^{1}\left(M, T_{M}\right)$ is equivalent to the classical Kuranishi versal deformation of $M$.

(iii) If $M$ is a Calabi-Yau manifold, then $\partial \equiv 0$ and the base of the above versal deformation can be canonically identified with the Barannikov-Kontsevich moduli space of (partially) extended complex structures.

Proof. The existence of a minimal versal deformation can be infered directly from Lemma 1.5.2 and Theorem 2.6. Nevertheless, we will give a detailed proof of this statement which makes all other claims, (i)-(iii), almost obvious. The idea of the proof is to show that, though the solution space, $\widehat{\mathrm{MC}}$, of Maurer-Cartan equations arising in our extended deformation theory is much larger than the solution space, MC, of the Maurer-Cartan equations in the original purely algebraic $\left(\Gamma\left(M, T_{M} \otimes \Omega_{M}^{0, \bullet}\right), \bar{\partial}\right)$-approach, the gauge group turns out to be much larger as well, and, crucially, at the level of quotients,

$$
\frac{\widehat{M C}}{\text { gauge group }}=\frac{M C}{\text { gauge group }},
$$

we have a canonical isomorphism.

Let $f:\left(\mathcal{X}, \partial_{\mathcal{X}}\right) \rightarrow\left(\mathcal{S}, \partial_{\mathcal{S}}, *\right)$ be a deformation of $(\mathcal{M}, \bar{\partial})$. We may assume without loss of generality that $\left(\mathcal{S}, \partial_{\mathcal{S}}, *\right)$ is dual to a differential Artin superalgebra $\left(A, \partial_{A}\right)$, cf. [Me2]. As in the proof of Proposition 2.4, we fix a local trivialization, $\phi: \mathcal{X} \simeq \mathcal{S} \times \mathcal{M}$, and decompose $^{2}$,

$$
\partial_{\mathcal{X}}=\partial_{\mathcal{M}}+\partial_{\mathcal{S}}+\Gamma
$$

where $\Gamma$ is an $f$-vertical odd holomorphic field $\Gamma$ vanishing at $\mathcal{M} \times *$. If $\left\{z^{\alpha}, z^{\dot{\alpha}}, \psi^{\dot{\alpha}}\right\}$ is a natural local coordinate system on $\mathcal{M},\left\{t^{i}\right\}$ a local coordinate system on $\mathcal{S}$ centered at $*$, then $\phi^{-1}$ maps the cartesian product of these into a local coordinate system on $\mathcal{X}$ in which $\Gamma$ can written as follows

$$
\Gamma=\sum_{\alpha=1}^{n} \Gamma^{\alpha} \frac{\partial}{\partial z^{\alpha}}+\sum_{\dot{\alpha}=1}^{n}\left(\Gamma^{\dot{\alpha}} \frac{\partial}{\partial z^{\dot{\alpha}}}+\gamma^{\dot{\alpha}} \frac{\partial}{\partial \psi^{\dot{\alpha}}}\right),
$$

for some local functions, $\Gamma^{\alpha}, \Gamma^{\dot{\alpha}}, \gamma^{\dot{\alpha}}$, of $\left\{z^{\alpha}, z^{\dot{\alpha}}, \psi^{\dot{\alpha}}, t^{i}\right\}$ which vanish at $t^{i}=0$. Hence, the local smooth map,

$$
\begin{aligned}
z^{\alpha} & \longrightarrow z^{\alpha}, \\
z^{\dot{\alpha}} & \longrightarrow z^{\dot{\alpha}}, \\
\psi^{\dot{\alpha}} & \longrightarrow \psi^{\dot{\alpha}}+\Gamma^{\dot{\alpha}}\left(z^{\beta}, z^{\dot{\beta}}, \psi^{\dot{\beta}}, t^{i}\right), \\
t^{i} & \longrightarrow t^{i},
\end{aligned}
$$

is invertable in a small open neighbourhood of $f^{-1}(*)$ in $\mathcal{X}$. It is easy to see that this transformation sends the component $\Gamma^{\dot{\alpha}} \partial / \partial z^{\dot{\alpha}}$ in $\Gamma$ to zero. Put another way, this component in $\Gamma$ can always be eliminated by an appropriate choice - "gauge" - of the trivialization $\phi$. Then the homology condition $\left[\partial_{\mathcal{X}}, \partial_{\mathcal{X}}\right]=0$ immediately implies that, in this gauge, $\gamma^{\dot{\alpha}}=0$ as well. (In physics jargon, both unwanted fields, $\Gamma^{\dot{\alpha}}$ and $\gamma^{\dot{\alpha}}$, correspond to purely 
gauge degrees of freedom, and, moreover, can be eliminated by one single gauge transform as above). Of the remaining coordinate transformations preserving the gauge, only the following ones,

$$
\begin{aligned}
z^{\alpha} & \longrightarrow z^{\alpha}+h^{\alpha}\left(z^{\beta}, z^{\dot{\beta}}, \psi^{\dot{\beta}}, t^{i}\right),\left.\quad h^{\alpha}\right|_{t=0}=0, \\
z^{\dot{\alpha}} & \longrightarrow z^{\dot{\alpha}} \\
\psi^{\alpha} & \longrightarrow \psi^{\dot{\alpha}} \\
t^{i} & \longrightarrow t^{i}
\end{aligned}
$$

do effectively change $\Gamma$. Now it is clear that, in the gauge $\Gamma^{\dot{\alpha}}=\gamma^{\dot{\alpha}}=0$, the solution space, $\widehat{\mathrm{MC}}$, of Maurer-Cartan equations in the deformation theory of extended complex manifolds,

$$
[\bar{\partial}, \Gamma]+\left[\partial_{\mathcal{S}}, \Gamma\right]+\frac{1}{2}[\Gamma, \Gamma]=0
$$

modulo the remaining gauge freedom $(G)$ can be canonically identified with the solution space of the Maurer-Cartan equations of the dg-Lie algebra $\left(\mathfrak{g}=\Gamma\left(M, T_{M} \otimes \Omega_{M}^{0, \bullet}\right), \bar{\partial}\right)$,

$$
\mathrm{MC}=\left\{\Gamma \in\left(\mathfrak{g} \otimes m_{A}\right)_{\tilde{1}} \mid \bar{\partial} \Gamma+\partial_{A} \Gamma+\frac{1}{2}[\Gamma, \Gamma]=0\right\},
$$

modulo the following gauge transformations,

$$
\Gamma \rightarrow \Gamma^{g}=e^{\operatorname{ad}_{g}} \Gamma-\frac{e^{a d_{g}}-1}{\operatorname{ad}_{g}}\left(d+\partial_{A}\right) g, \quad \forall g \in\left(\mathfrak{g} \otimes m_{A}\right)_{\tilde{0}} .
$$

Here $m_{A}$ stands for the maximal ideal in the Artin superalgebra $A$. It is proved in Me2 that the associated deformation functor

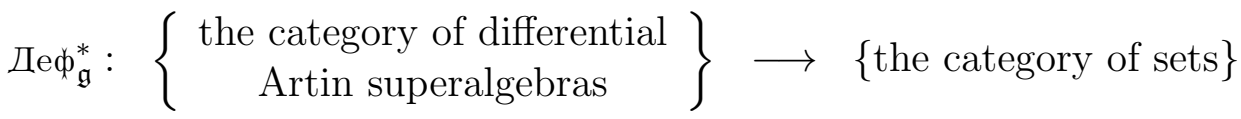

$$
\begin{aligned}
& \left(A, \partial_{A}\right) \quad \longrightarrow \quad \mathrm{MC} / \text { gauge group }
\end{aligned}
$$

is always unobstructed, and can be versally represented by $\left(\mathbf{H}^{*}\left(M, T_{M}\right), \partial, 0\right)$, where the homological vector field $\partial$ is an invariant of $\mathfrak{g}$. (The latter was called in Me2 Chen's vector field as its origin can be traced back to Chen's power series connection in the theory of iterated integrals.)

With the established isomorphism of versal moduli spaces, the statement 2.7.2(iii) becomes an obvious corollary of Lemma 2.1 in [BK].

It remains to check the existence of the classical limit 2.7.2(ii). Picking up a Hermitian metric on $M$, we can construct the adjoint, $\bar{\partial}^{*}$, of the Dolbeault operator $\bar{\partial}$ on $\mathfrak{g}$, the Laplacian $\square=\bar{\partial} \bar{\partial}^{*}+\bar{\partial}^{*} \bar{\partial}$, the Green function $G$, and we can identify the cohomology space $\mathbf{H}^{\bullet}\left(M, T_{M}\right)$ with the space of harmonic elements, Ker $\bar{\partial} \cap \operatorname{Ker} \bar{\partial}^{*}$, in $\mathfrak{g}$. Choosing next a harmonic basis, $e_{i}$, in $\mathbf{H}^{\bullet}\left(M, T_{M}\right)$, and denoting the associated linear coordinates by $t^{i}$ we can represent the Chen's vector field, $\partial=\sum_{i} \partial^{i}(t) \partial / \partial t^{i}$, as follows Me2

$$
\sum_{i} \partial^{i}(t) e_{i}=-\frac{1}{2} P[\Gamma, \Gamma]
$$


where $P: \mathfrak{g} \rightarrow \mathbf{H}^{\bullet}\left(M, T_{M}\right)$ is the natural projection to the harmonic constituent, and $\Gamma=\bigoplus_{n=1}^{\infty} \Gamma_{[n]}$ is given by a recursive formula,

$$
\begin{aligned}
\Gamma_{[1]} & =\sum_{i} t^{i} e_{i} \\
\Gamma_{[2]} & =-\frac{1}{2} G \bar{\partial}^{*}\left[\Gamma_{[1]}(t), \Gamma_{[1]}(t)\right], \\
\Gamma_{[3]} & =-\frac{1}{2} G \bar{\partial}^{*}\left(\left[\Gamma_{[1]}(t), \Gamma_{[2]}(t)\right]+\left[\Gamma_{[2]}(t), \Gamma_{[1]}(t)\right]\right), \\
\ldots & \\
\Gamma_{[n]} & =-\frac{1}{2} G \bar{\partial}^{*}\left(\sum_{k=1}^{n-1}\left[\Gamma_{[k]}(t), \Gamma_{[n-k]}(t)\right]\right) \\
\ldots &
\end{aligned}
$$

It is now obvious that Zeros $(\partial) \cap \mathbf{H}^{1}\left(M, T_{M}\right)$ is precisely the Kuranishi analytic subspace [K]. Moreover, the fibres, $f^{-1}(t)$, of our minimal versal deformation $f$ over this subspace are precisely the extended complex manifolds, $\left(\mathcal{M}_{t}, \bar{\partial}_{t}\right)$, associated, via the construction 1.5 , to the usual complex manifolds $M_{t}$ lying over $t$ in the classical Kuranishi versal deformation of $M$.

Acknowledgement. This work was done during author's visit to the Max Planck Institute for Mathematics in Bonn. Excellent working conditions in the MPIM are gratefully acknowledged. I would like to thank Yu.I. Manin for many discussions.

\section{References}

[Ba] S. Barannikov, Generalized periods and mirror symmetry in dimensions $n>3$, preprint math/9903124.

[BK] S. Barannikov and M. Kontsevich, Frobenius manifolds and formality of Lie algebras of polyvector fields, Internat. Math. Res. Notices, no.4 (1998), 201-215.

[GM] W.M. Goldman and J.J. Millson, The deformation theory of representations of fundamental groups of compact Kähler manifolds, Inst. Hautes Etudes Sci. Publ. Math. 67 (1988), 43-96.

[K] K. Kodaira, Complex manifolds and deformations of complex structures, Springer 1986.

[Ko1] M. Kontsevich, Homological algebra of mirror symmetry, Proceedings of the International Congress of Mathematicians, Vol.1 (Zürich, 1994) (Bürkhäuser, Basel), 1995, pp. 120-139.

[Ko2] M. Kontsevich, Deformation quantization of Poisson manifolds I, math/9709040.

[Ma1] Yu.I. Manin, Frobenius manifolds, quantum cohomology, and moduli spaces, AMS, Providence, 1999.

[Ma2] Yu.I. Manin, Moduli, Motives, Mirrors and other Marvels, lectures in the MPIM, Bonn, winter-spring 2000.

[Me1] S.A. Merkulov, The extended moduli space of special Lagrangian submanifolds, Commun. Math. Phys. 209 (2000), 13-27. 
[Me2] S.A. Merkulov, Frobenius $\infty$ invariants of homotopy Gerstenhaber algebras, I, Duke Math. J. (2000), to appear.

[P] A. Polishchuk, Homological mirror symmetry with higher products, preprint math/9901025.

[PZ] A. Polishchuk and E. Zaslow, Categorical mirros symmetry: the elliptic curve, Adv. Theor. Math. Phys. 2 (1998), 443-470.

Max Planck Institute for Mathematics in Bonn, and Department of Mathematics, University of Glasgow sm@maths.gla.ac.uk 